normally and their weight is artificial. Most patients lose weight after sanatorium treatment even if the disease is arrested. Having reached the normal weight for the stem length and chest measurement they maintain this weight if the disease is arrested, and then the relationship between weight and V.C. is found. During the loss of weight owing to increasing disease or the gain of weight during forced feeding and rest, however, there is no reliable relationship with V.C. The significance of weight is seen when patients after treatment have returned to their normal lives; a normal weight maintained is a good omen, but steady loss of weight indicates that the disease is spreading.

Conclusions.

The conclusions at which we arrived in 1920 seem more than justified now after a further two years study of the cases. ${ }^{6}$

1. In cases of pulmonary tuberculosis there is a definite decrease in V.C. But after treatment the V.C. may improve, and as in one of our cases (No. 304) actually become normal, in spite of the fact that the disease later broke out again and proved fatal.

2. An improvement in the clinical condition of the patient is found to be accompanied by an increased V.C., whilst an advance of the disease results in a decreased V.C.

3. The determination of the T.C. is useful for the classification of cases of pulmonary tuberculosis, because it is possible by this means numerically to express the injury to health (e.g., deoree of toxæmia and amount of lung diseased) which otherwise would depend on the individual interpretation of physical sions by different observers. In this connexion it should be noted that classification by physical signs alone may place a patient nearing death from an acute lesion in a high category, whilst another patient, with satisfactory fibrosis and extensive cavitation, likely to live for several years, may be placed in the lowest category. Dr. Cameron criticises this conclusion and rightly points out that the V.C. may be low, not only because of toxæmia, but also because of extensive fibrosis, and thinks that V.C. alone is of very restricted value and should be taken in conjunction with physical signs. We agree with Dr. Cameron if one reading alone is taken, and are of opinion that one reading alone is always of restricted value. The importance lies in taking repeated readings, as Dr. Cameron himself shows. For instance, one of us (L. S. T. B.) from London used to visit at Oxford the other (G. D.) who knew nothing whatever about the condition of the patient. If the percentage readings presented to him were say: $-40,-39 \cdot 6,-41,-40 \cdot 4,-39 \cdot 8,-40 \cdot 8$, over a period of six months, he would at once say, "Chronic case holding his own" ; on the other hand, if the readings were $-26,-30,-29,-38,-46,-48$ he would say: "This patient is dying." So impressed were we by the accuracy of these findings that one of us (L.S. T. B.) published a classification based largely on V.C.

4. As an aid to diagnosis even a single, but more particularly a repeated examination of the V.C. of doubtful cases will also prove useful. If a normal V.C. is found, this patient is most unlikely to be suffering from pulmonary tuberculosis, whereas if the V.C. is much decreased this patient should be suspected, though it must always be borne in mind that other diseases may also cause a lowering of the V.C.

5. Finally a systematic study of the V.C. in its proper relationship to body size has given important information as to the beneficial effects of different treatments of pulmonary tuberculosis.

After many thousand observations we have seen no ill-effect from taking V.C. We note that Dr. Cameron after 6000 readings has found no ill-effect, and we fail to see why it should be harmful. It is, in our opinion, far less strain to the patient than even a mild attack of coughing.

- Since this report was prepared a paper by J. A. Myers with similar conclusions, entitled "Comparison of Vital Capacity Readings and X Ray Findings in Pulmonary Tuberculosis," has appeared. Amer. Rev. Tub., 1922, ซ., 884 .

\section{THE BED ISOLATION OF CASES OF INFECTIOUS DISEASE.}

\section{FURTHER EXPERIENCE AT FAZAKERLEY}

By C. RUNDLE, O.B.E., M.D. LoND., D.P.H.

MEDICAL SUPERINTENDENT, FAZAKERLEY CITY HOSPITALS AND SANATORIUI, IIVERPOOL.

DURING the period February, 1920, to June, 1922, the following cases of infectious disease have been treated in one bed-isolation ward of 26 beds at the Fazakerley Isolation Hospital :-

\section{Measles}

Chicken-pox

Diphtheria

Whooping-cough . .

Scarlet fever

Mumps

Cerebrospinal fever

German measles

Acute ant. poliomyelitis.

Encephalitis lethargica.

Typhoid fever

Scabies

\begin{tabular}{r|llll}
108 & Measles with- & & \\
45 & Whooping-cough & $\ldots$ & 4 \\
19 & Diphtheria & $\ldots$ & $\ldots$ & 3 \\
20 & Chicken-pox & $\ldots$ & $\ldots$ & 2 \\
56 & Scarlet fever with- & & \\
Chicken-pox & $\ldots$ & $\ldots$ & 1 \\
$\mathbf{5}$ & Whooping-cough & $\ldots$ & 1 \\
$\mathbf{1 4}$ & Diphtheria & $\ldots$ & $\ldots$ & 1 \\
Mumps . & Whooping-cough with- & $\ldots$ & 1 \\
2 & Chicken-pox & $\ldots$ & $\ldots$ & 3 \\
6 & German measles & $\ldots$ & 1 \\
29 & Erysipelas & $\ldots$ & $\ldots$ & 80 \\
1 & Anthrax .. & $\ldots$ & $\ldots$ & 2
\end{tabular}

Of this total one case only developed a secondary infection-namely, a patient admitted with whoopingcough on Dec. 8th, 1921, who developed chicken-pox on Jan. 29th, 1922. The circumstances under which these patients were admitted to a bed-isolation ward were similar to those laid down in a previous report. I

The cases were admitted in all stages of infection, they were chiefly of susceptible ages, and they include under the group " other diseases" a large number of conditions not commonly included amongst the acute infections-e.g., tonsillitis, pneumonia, and tuberculosis.

The routine observed for the avoidance of secondary infection is shown in the following transcript from the printed notice hung up in the ward:-

\section{BED ISOLATION AT THE CITY HOSPITAL,} FAZAKERLEY.

Precautions to be Observed.

CoAts to be worn for every purpose, and hands washed (using nail-brush) after their removal.

Feeding and drinking utensils to be rinsed and placed, immediately after use, into the kitchen steriliser. They must not be laid down in the sinks in the kitchen or elsewhere Coats must not be worn in the kitchen, but the nurse should return to the bedside bowl for washing purposes after taking out the feeding utensils, \&c.

In wards, other than "G " and " $\mathrm{C}$," bed-isolation crockery must be distinctive, or separately marked.

Separate and marked sanitary utensils, lavatory cloths, bath blankets.

Coat must be worn when removing clothing, bedding, sanitary utensils, \&c., to the lavatories; the coat to be subsequently removed at the bedside and hands disinfected. Separate washing-bowl, towel, nail-brush, and thermometer to be used in cases of chicken-pox, measles, enteric fever, and in any case where the treatment is likely to be frequent or prolonged.

Patients not to be allowed to mix with other patients, sit at common table, or use lavatories.

Bedside bowls to be immediately emptied and refilled after use. For this purpose the nurse must not wear coat to lavatory. The coat must be removed and hands washed, using nail-brush, before refilling bowl.

All temperatures to be taken in axilla, and thermomete washed in lysol after use.

No toys, books, or papers to be allowed.

The doctor will decide which patients are to undergo bed isolation, but all patients admitted to the bed isolation wards are to be so dealt with from the moment of admission until the doctor has visited the wards and given his instructions in the case. The only exception to this rule is erysipelas, which is to be treated with ordinary cleanliness only.

No nurse engaged in the active treatment of puerperal fever is to attend, in any way, upon a case of erysipelas or scarlet fever. Rubber gloves (reserved for the purpose) must be used in vaginal douching.

${ }^{1}$ C. Rundle and A. H. G. Burton: THE LANCET, 1912, i., 720. 